\title{
A New Radiation Condition for Ships Travelling with Very Low Forward Speed
}

\author{
*Zhi-Ming Yuan, Atilla Incecik, Laibing Jia \\ Department of Naval Architecture, Ocean and Marine Engineering, University of Strathclyde, Henry Dyer Building, G4 0LZ, Glasgow, UK
}

\begin{abstract}
The 3-D Rankine source method is becoming a very popular method for the seakeeping problem, especially when marine vessels are travelling with forward speed. However, this method requires an accurate radiation condition to ensure that the waves propagate away from the ship. Considerable effort has been expanded for the accurate formulation of the radiation condition when a ship travels at the moderate to high speed range, and significant progress has been made in the last few decades when the Brard number $\tau$ is greater than 0.25 . But for the very low forward speed problem $(\tau<0.25)$, the ordinary radiation condition will not be valid since the scattered waves will propagate ahead of the vessel. The primary purpose of the present study which is to be presented in this paper is to investigate the formulation of a new radiation condition which can account for the very low forward speed problem. To verify this new radiation condition, a Wigley III hull has been modelled by using the 3-D panel method. The hydrodynamic coefficients and the wave pattern of the vessel at zero speed and travelling with very low and medium forward speeds are calculated separately. From the comparisons of the present results with experimental measurements, it can be concluded that the new radiation condition can accurately predict the hydrodynamic properties of vessels travelling with low to medium forward speed or stationary in waves.
\end{abstract}

Keywords: Low forward speed; Rankine source method; Radiation condition; Wave pattern; Boundary element method.

\section{Introduction}

The prediction of hydrodynamic responses of marine structures with forward speed has drawn intensive attention for many decades. There are a considerable number of publications concerning the prediction of hydrodynamic responses of vessels travelling in waves. Based upon the Green function employed in the boundary integral formulation, these papers could be divided into two categories. In the first category, the translating and pulsating sources are only distributed on the wetted body surface. This method utilizes a Green function that satisfies the Kelvin free surface conditions, as well as the radiation condition (Newman, 1985, 1992). It is an effective method for the zero forward problems, but if the vessel is travelling with forward speed, this method still has some limitations. Firstly, it could not account for the near-field flow condition. Although some researchers (Lee and Sclavounos, 1989; Nossen et al., 1991) extended it to include the near-field free surface condition, the so-called irregular frequency still cannot be avoided. And it will bring singularity to the coefficient matrix equation. Secondly, it is impossible for the Green function to account for the interaction between the steady and unsteady flow.

Corresponding author at: Dep. of Naval Architecture, Ocean \& Marine Engineering, University of Strathclyde.

Henry Dyer Building, G4 0LZ, Glasgow, UK.

Tel: + 44 (0)141548 2288. Fax: +44 (0)141552 2879.

E-mail address: zhiming.yuan@strath.ac.uk 
The second category is called Rankine source approach, which uses a very simple Green function in the boundary integral formulation. This method requires the sources distributed not only on the body surface, but also on the free surface and control surface. Therefore, a flexible choice of free-surface conditions can be realized in these methods. The coupled behavior between steady and unsteady wave potential could be expressed in a direct formula. Meanwhile, the nonlinearity on the free surface could also be added in the boundary condition. For these reasons, the Rankine source approach has been used by many investigators since it has been first proposed by Hess and Smith (Hess and Smith, 1964). Investigators from MIT (Kring, 1994; Nakos and Sclavounos, 1990; Scalvounos and Nakos, 1988) applied the Rankine source approach to model steady and unsteady waves as a ship moves in waves. An analysis technique developed by Sclavounos and Nakos (Scalvounos and Nakos, 1988) for the propagation of gravity waves on a panelized free surface showed that a Rankine method could adequately predict the ship wave patterns and forces. Their work led to the development of a frequency-domain formulation for ship motions with a consistent linearization based upon the double body steady flow model which assumes small and moderate Froude numbers. Applications were reported by Nakos et al. (1990). This model was extended to the time domain by Kring (1994) who also proposed a physically rational set of Kutta conditions at a ship's transom stern. Recently, Gao et al. (Gao and Zou, 2008) developed a high-order Rankine panel method based on NonUniform Rational B-Spline (NURBS) to solve the 3-D radiation and diffraction problems with forward speed. Their results had very good agreement with the experimental data.

However, there are still some limitations for the extensive use of the Rankine source approach. First of all, the Rankine source method requires much more panels which will considerably increase the computation time, especially when the matrix equation is full range matrix. However, the computation time will strongly depend on the numerical method and computer language. As the performance of computers increase rapidly, it only takes less than 1 minute to solve a $10^{4 \times 10^{4}}$ full range matrix using Matlab. Typically, the number of panels will be no more than 10, 000. The computation time is acceptable in engineering applications. Besides, the Rankine source method requires a suitable radiation boundary condition to account for the scattered waves in current. A very popular radiation condition for the forward speed problem, which is so-called upstream radiation condition, was proposed by Nakos (Nakos, 1990). The free surface was truncated at some upstream points, and two boundary conditions were imposed at these points to ensure the consistency of the upstream truncation of the free surface. Another method to deal with the radiation condition is to move the source points on the free surface at some distance downstream (Jensen et al., 1986). The results from these two methods show very good agreement with published experimental data when the Brard number $\tau(\tau=\mathrm{u} \omega / \mathrm{g})$ is greater than 0.25 , since they are both based on the assumption that there is no scattered wave travelling ahead of the vessel. However, when the forward speed of the vessel is very low, the Brard number will smaller than 0.25 . When this case occurs, the scattered waves could travel ahead of the vessel, and these traditional radiation conditions could no longer be valid.

Low forward speed problem could occur when the ships are travelling in harbour areas or between tugs and vessels during escorting or manoeuvring and berthing operations as well as during ship-to-ship operations for cargo transfers during oil and gas offloading operations. It also occurs in transport and towage of the offshore structures to their operation sites. Coudray and Le Guen (1992) compared the wave patterns generated by a submerged source for various simplifications of the linearized Neumann-Kelvin boundary condition at low value of $\tau=0.128$. Ba and Guillbaud (1995) used Kelvin singularities to get a fast 
and efficient method to compute the unsteady Green's function for different Froude numbers. They compared their wave patterns with that of Noblesse and Hendrix (1992) at various values of $\tau$ ranging from 0.2 to 4 and good agreement was achieved. However, these studies were all based on the Green function which could satisfy the far field radiation condition automatically. The low forward speed problem can also be solved by using Rankine source panel method, but the main difficult is due to the numerical satisfaction of the radiation condition. To find a reasonable radiation condition for very low forward speed problem, Das and Cheung (2012a, b) provided an alternate solution to the boundary-value problem for forward speeds above and below the group velocity of the scattered waves. They corrected the Sommerfeld radiation condition by taking into account the Doppler shift of the scattered waves at the control surface that truncates the infinite fluid domain. They compared their results with the experimental data, and good agreement was achieved.

In the present study, this new radiation condition of Das and Cheung (2012b) is adapted to complement of the boundary-value problem. A Wigley hull with different forward speeds will be studied. The results obtained are compared with Journee's experimental data (Journee, 1992), which could verify the effectiveness of the new radiation condition.

\section{Mathematical formulations based on potential theory}

\subsection{Boundary value problem}

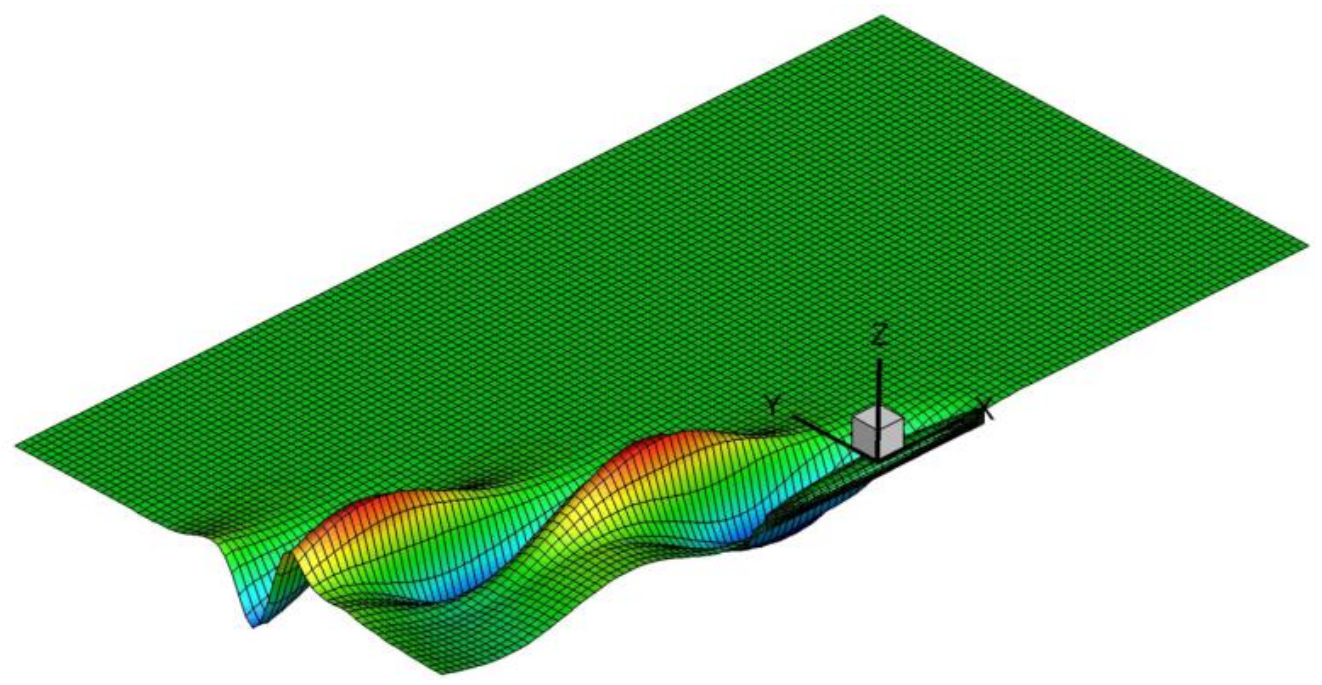

Fig. 1 An example vessel and coordinate system

Fig. 1 shows a vessel travelling with a constant forward speed in a Cartesian coordinate system which is moving together with the body. The origin is located on the still water and axis $\mathrm{Z}$ points upward. $\mathrm{X}$ and $\mathrm{Y}$ axis is on the geometric centroid of the water-plane. Based on the assumption that the surrounding fluid is inviscid and incompressible, and that the motion is irrotational, the total velocity potential exists which satisfies the Laplace equation in the whole fluid domain. For linearization, the total potential can be decomposed into 


$$
\Psi(\vec{x}, t)=u_{0} x+\varphi_{s}(\vec{x})+\operatorname{Re} \sum_{j=0}^{7} \eta_{j} \varphi_{j}(x, y, z) e^{-i \omega_{e} t}
$$

where $\varphi_{s}$ is the steady potential and it is assumed to be small. $\varphi_{j}(j=1,2, \ldots, 6)$ is the radiation potential corresponding to the oscillations of the body in six degrees of freedom and $\eta_{j}(j=1,2, \ldots, 6)$ is the corresponding motion amplitude ( $\eta_{1}$ : surge; $\eta_{2}$ : sway; $\eta_{3}$ : heave; $\eta_{4}:$ roll; $\eta_{5}:$ pitch; $\eta_{6}:$ yaw). $\varphi_{0}$ is potential of the incident waves and $\eta_{0}$ is the incident wave amplitude. $\varphi_{7}$ is the diffraction potential due to the diffraction wave amplitude $\eta_{7}$ with $\eta_{7}=\eta_{0}$ and $\omega_{e}$ is the encounter frequency, which can be written as

$$
\omega_{e}=\omega-u_{0} k \cos \beta
$$

Linear wave theory provides the potential for unit-amplitude incident waves as

$$
\varphi_{0}=-\frac{i g}{\omega_{0}} e^{k[z+i(x \cos \beta+y \sin \beta)]}
$$

where $k=\omega_{0}^{2} / g$, $\omega_{0}$ is the incident wave frequency, $\beta$ is the angle of wave heading $(\beta=180 \mathrm{deg}$ corresponds to head sea).

When the steady wave flow is selected as a basic flow model, the steady perturbation potential $\varphi_{s}$ can then be solved by the following boundary value problem:

$$
\begin{gathered}
\nabla^{2} \varphi_{s}=0 \quad \text { in the fluid domain } \\
u_{0}^{2} \frac{\partial^{2} \varphi_{s}}{\partial x^{2}}+g \frac{\partial \varphi_{s}}{\partial z}=0 \quad \text { on the undisturbed free surface } S_{f} \\
\frac{\partial \varphi_{s}}{\partial n}=u_{0} n_{1} \quad \text { on the mean wetted part of the body surface } S_{b}
\end{gathered}
$$

Moreover, a radiation condition in the far field control surface must be imposed to prevent the wave reflection from the truncated computational domain. This will be discussed in the next section.

The unsteady perturbation potential $\varphi_{j}$ can then be solved by the following boundary value problem:

$$
\begin{gathered}
\nabla^{2} \varphi_{j}=0 \quad \text { in the fluid domain } \\
-\omega_{e}^{2} \varphi_{j}-2 i \omega_{e} u_{0} \frac{\partial \varphi_{j}}{\partial x}+u_{0}^{2} \frac{\partial^{2} \varphi_{j}}{\partial x^{2}}+g \frac{\partial \varphi_{j}}{\partial z}=0 \quad \text { on the undisturbed free surface } S_{f} \\
\frac{\partial \varphi_{j}}{\partial n}=\left\{\begin{array}{cc}
-i \omega_{e} n_{j}+u_{0} m_{j}, j=1,2, \ldots, 6 \\
-\frac{\partial \varphi_{0}}{\partial n}, j=7 & \text { on the mean wetted part of the body surface } S_{b}
\end{array}\right.
\end{gathered}
$$


The radiation condition at infinity is also imposed to complete the boundary value problem, which will be discussed latter. The generalized normal vectors are defined as

$$
n_{j}=\left\{\begin{array}{c}
\vec{n}, j=1,2,3 \\
\vec{x} \times \vec{n}, j=4,5,6
\end{array}\right.
$$

and $\vec{n}=\left(n_{1}, n_{2}, n_{3}\right)$ is the unit normal vector directed inward on body surface $S_{b}, \vec{x}=(x, y, z)$ is the position vector on $S_{b}$. The $m_{j}$ denotes the $j$-th component of the so-called $m$-term, which can be expressed as

$$
m_{j}=\left\{\begin{array}{c}
-(\vec{n} \cdot \nabla) \nabla \varphi_{s}, j=1,2,3 \\
-(\vec{n} \cdot \nabla)\left(\vec{x} \times \nabla \varphi_{s}\right), j=4,5,6
\end{array}\right.
$$

The $m$-terms provide coupling effects between the steady and unsteady flows and involve the second derivatives of the steady potential. However, in the present study, we are interested in the very low forward speed problem. Therefore, the Neumann-Kelvin linearization can be used to simplify the m-terms,

$$
\begin{aligned}
& \left(m_{1}, m_{2}, m_{3}\right)=(0,0,0) \\
& \left(m_{4}, m_{5}, m_{6}\right)=\left(0, n_{3},-n_{2}\right)
\end{aligned}
$$

\subsection{Radiation condition}

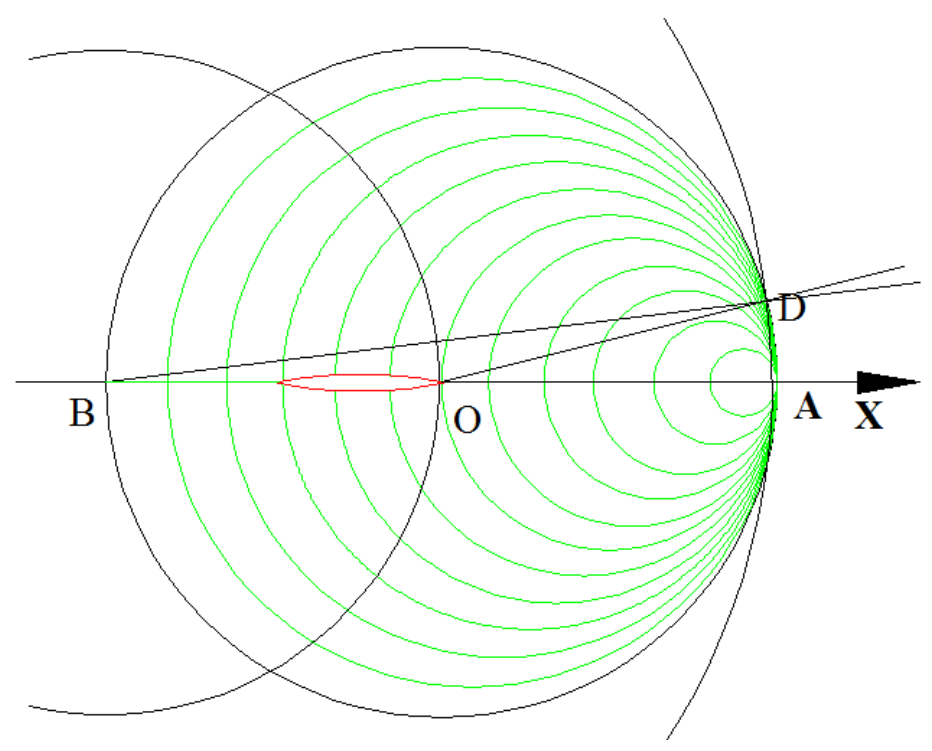

Fig. 2 Sketch of Doppler shift 


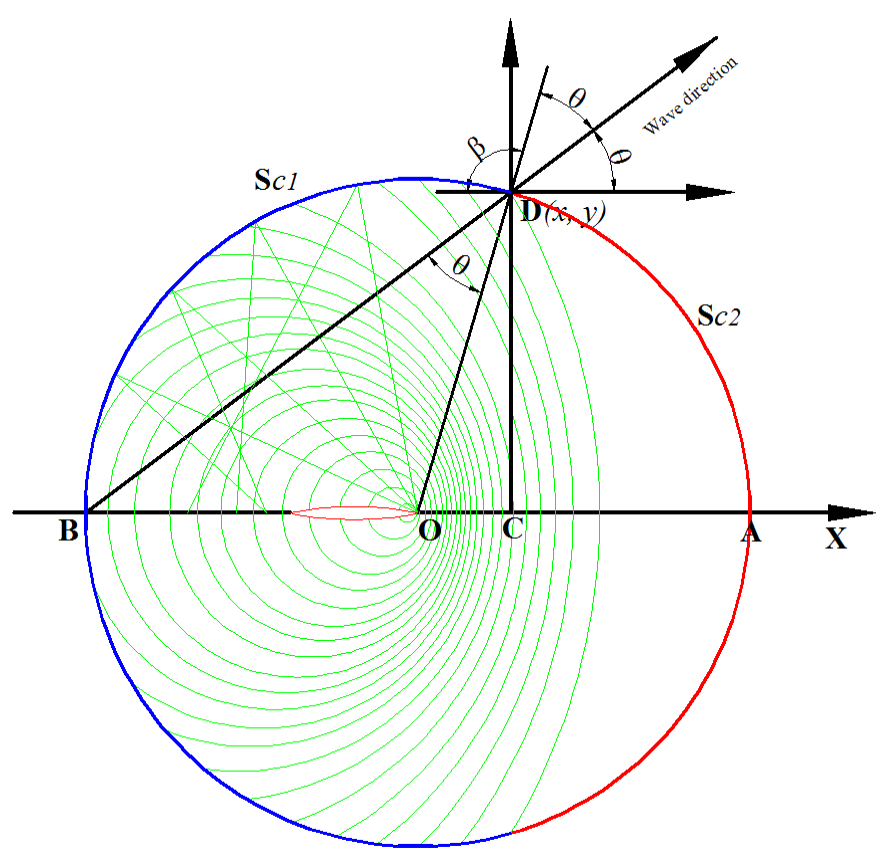

Fig. 3 Radiation condition

Fig. 2 shows the Doppler Shift of scattered wave field by a vessel travelling with constant forward speed $u_{0}$ in the positive $x$ direction. When a vessel is moving from point $B$ to point $O$, the traveling time should be $t=B O / u_{0}$. During this period of time, the vessel produces scattered waves all along $B O$ (the first scattered wave should arise at point $\mathrm{B}$ ). The control surface here is defined as a circle with its centroid on point $O$ and its radius as $B O$. If $u_{0}$ is small, the travelling time $t$ will be very long, which means the scattered waves produced at point $B$ can travel a long distance. When it just reaches point $A$, the wave group is reaching point $O$ (the wave group velocity is half of scattered wave velocity). In this case, the Brard number $\tau=0.25$ $\left(\tau=u_{0} \omega / g\right), u_{0}=u_{c}\left(u_{c}\right.$ denotes the critical forward speed of the vessel at $\left.\tau=0.25\right)$ and the scattered wave can reach any points on the control surface. If $\tau<0.25$ and $u_{0}<u_{c}$, the wave group will go head of the vessel. Therefore, the upstream radiation condition could not be used any longer. If $\tau>0.25$ and $u_{0}>u_{c}$, the scattered wave can only get to point $D$, as shown in Fig. 3. On arc $D A$, there are no scattered waves reaching here, and it is defined as $S_{C 2}$. To the contrary, arc $B D$ is defined as $S_{C 1}$, on which a different radiation condition should be imposed. From Fig. 3, the wave direction of a scattered wave reaching point $D(x, y)$, which is produced at $B$, have been rotated by an angle $\theta$ (if there is no forward speed, the wave direction should be along $O D$. The velocity of the scattered wave is defined as $c, B O / u_{0}=B D / c$. According to the sine theorem, it can be easily transferred to

$$
\frac{u_{0}}{c}=\frac{\sqrt{x^{2}+y^{2}}}{y} \sin \theta
$$

The scattered wave velocity at $D$ can be expressed as

$$
c=\frac{\omega_{s}}{k_{s}}
$$


where $\omega_{s}$ is the angular frequency of the scattered waves from a fixed reference point given as

$$
\omega_{s}=\omega_{e}+u_{0} k_{s} \cos \left[\tan ^{-1}\left(\frac{y}{x}\right)-\theta\right]=\sqrt{g k_{s} \tanh k_{s} d}
$$

in which $k_{s}$ is the local wave number at $D(x, y)$, and $d$ is the water depth.

Once the coordinates of any arbitrary point on the control surface are given, the unknowns $\theta$ and $k_{s}$ could be obtained by solving the nonlinear equation system (13)-(15). If one cannot find solutions, these points must be on the control surface $S_{c 2}$. Otherwise, they are on $S_{c 1}$. The radiation condition is defined as two different equations,

$$
\begin{gathered}
\frac{\partial \varphi_{j}}{\partial n}-i k_{s} \varphi_{j} \cos \theta=0 \quad(j=1,2, \ldots, 6) \text { on } S_{c 1} \\
\nabla \varphi_{j}=0 \quad(j=1,2, \ldots, 6) \text { on } S_{c 2}
\end{gathered}
$$

Eq. (16) is an updated Sommerfeld radiation condition with forward speed correction. If the forward speed is zero, $k_{s}=k, \theta=0$ and Eq. (16) could reduce to the Sommerfeld radiation condition

$$
\frac{\partial \varphi_{j}}{\partial n}-i k \varphi_{j}=0 \quad(\mathrm{j}=1,2, \ldots, 6) \quad \text { on } S_{c}
$$

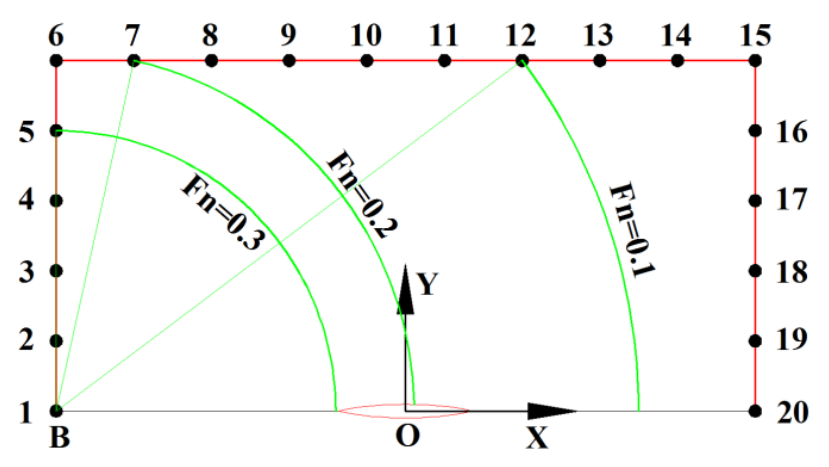

Fig. 4 Rectangle control surface

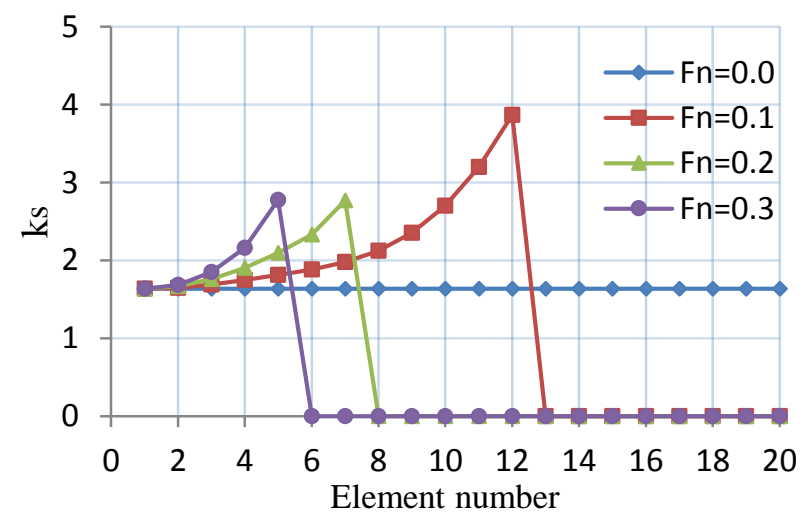


Fig. 5 Local wave number on rectangle control surface

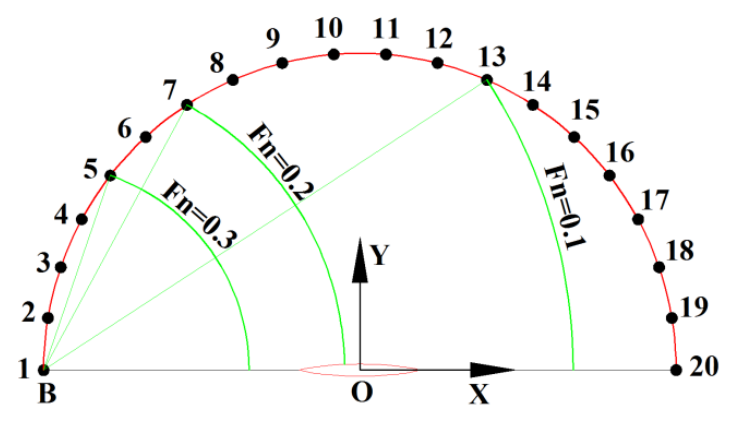

Fig. 6 Circular control surface

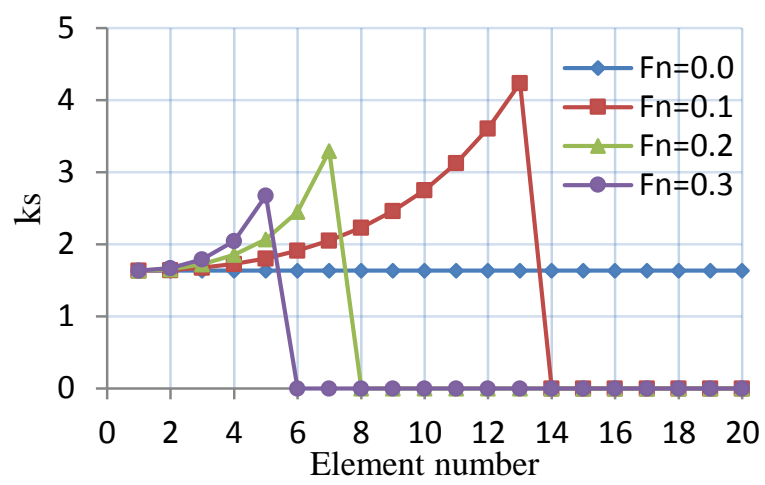

Fig. 7 Local wave number on circular control surface

Fig. 4 and Fig. 6 show a Wigley hull traveling with different Froude number in the positive $x$ direction and the free surface is truncated by a rectangle and circular control surface respectively. The control surface is dispersed into a series of discrete elements (20 elements in the present model). Fig. 5 and Fig. 7 are the results of the local wave number $k_{s}$ on different control surfaces and different Froude numbers. $k_{s}=0$ illustrates that no solution is found at these elements and these points are on $S_{C 2}$. It can be found from Fig. 5 that as the Froude number increases, the range of $S_{c 1}$ shrinks. At $F n=0, \theta=0$ and $k_{s}=k$, which is a constant independent of the location. When $F n=0.1$, the scattered wave at $B$ can only reach No.1-12 elements, and for No.13-20 elements, no solution can be found here. When the Froude number increases to 0.3, only No.1-5 elements on the downstream end can be influenced by the scattered waves. The values of $k_{s}$ are inversely proportional to the distance between $B$ and the points on the control surface. Fig. 7 transmits the same information as Fig. 5, which indicates that the truncation of free surface could be arbitrary and $k_{s}$ and $\theta$ are only determined by the coordinates of the points on the control surface. However, in the numerical study, the free surface range will greatly influence the computational results, and this will be discussed in the next section.

\subsection{Equations of motion}

Once the unknown potential $\varphi_{s}$ and $\varphi_{j}$ are solved, the steady pressure and the time-harmonic pressure can be obtained from Bernoulli's equation: 


$$
\begin{gathered}
p_{s}=\rho\left(u_{0} \frac{\partial \varphi_{s}}{\partial x}-\frac{1}{2} \nabla \varphi_{s} \cdot \nabla \varphi_{s}\right) \\
p_{j}=-\rho\left[i \omega_{e} \varphi_{j}+\nabla\left(\varphi_{s}+u_{0} x\right) \cdot \nabla \varphi_{j}\right], \quad j=0,1, \ldots, 7
\end{gathered}
$$

The steady hydrodynamic forces are obtained by the pressure integral on the wetted body surface as follows:

$$
F_{i}^{s}=\iint_{S_{b}} p_{s} n_{i} d S, \quad i=1,2, \ldots, 6
$$

The wave making resistance can be defined as

$$
C_{w}=\frac{F_{1}^{s}}{\frac{1}{2} \rho u_{0}^{2} S}
$$

where $S$ is the area of the wetted body surface. The hydrodynamic force produced by the oscillatory motions of the vessel in the six degrees of freedom can be derived from the radiation potential as

$$
F_{i}^{D}=\sum_{j=1}^{6} \iint_{S_{b}} p_{j} n_{i} d S \cdot \eta_{j}=\sum_{j=1}^{6}\left[\omega_{e}^{2} \mu_{i j}+i \omega_{e} \lambda_{i j}\right] \eta_{j}, \quad i=1,2, \ldots, 6
$$

where $\mu_{i j}$ and $\lambda_{i j}$ are the added mass and damping coefficients matrices respectively, which can be written as

$$
\begin{aligned}
& \mu_{i j}=\frac{1}{\omega_{e}^{2}} \iint_{S_{b}}\left(u_{0} \frac{\partial \varphi_{R j}}{\partial x}-\omega_{e} \varphi_{I j}\right) n_{i} d s, \quad i, j=1,2, \ldots, 6 \\
& \lambda_{i j}=-\frac{1}{\omega_{e}} \iiint_{S_{b}}\left(u_{0} \frac{\partial \varphi_{I j}}{\partial x}+\omega_{e} \varphi_{R j}\right) n_{i} d s, \quad \mathrm{i}, \mathrm{j}=1,2, \ldots, 6
\end{aligned}
$$

$\varphi_{R j}$ is the real part of $j$-th potential, and $\varphi_{I j}$ is the imaginary part. The wave excitation force can be obtained by the integration of incident and diffraction pressure as

$$
F_{i}^{e x t}=\iint_{S_{b}}\left(p_{0}+p_{7}\right) n_{i} d S \cdot \eta_{0}
$$

Applying Newton's second law, the six components of ship motions in the frequency domain can be obtained by solving the following equations of motions: 


$$
\sum_{j=1}^{6}\left[-\omega_{e}^{2}\left(M_{i j}+\mu_{i j}\right)+i \omega_{e} \lambda_{i j}+K_{i j}\right] \eta_{j}=F_{i}^{e x t}
$$

The mass matrix $M_{i j}$ and restoring matrix coefficient $K_{i j}$ are given by

$$
\begin{gathered}
M_{i j}=\left[\begin{array}{cccccc}
m & 0 & 0 & 0 & m z_{G} & 0 \\
0 & m & 0 & -m z_{G} & 0 & m x_{G} \\
0 & 0 & m & 0 & -m x_{G} & 0 \\
0 & -m z_{G} & 0 & I_{44} & 0 & I_{46} \\
m z_{G} & 0 & -m x_{G} & 0 & I_{55} & 0 \\
0 & m x_{G} & 0 & I_{64} & 0 & I_{66}
\end{array}\right] \\
K_{i j}=\left[\begin{array}{ccccccc}
0 & 0 & 0 & 0 & 0 & 0 \\
0 & 0 & 0 & 0 & 0 & 0 \\
0 & 0 & \rho g A_{w} & 0 & 0 & 0 \\
0 & 0 & 0 & \rho g\left(I_{w 1}+V z_{B}\right) & 0 & 0 \\
0 & 0 & -\rho g M_{w} & 0 & 0 g\left(I_{w 2}+V z_{B}\right) & 0 \\
0 & 0 & 0 & 0 & 0 & 0
\end{array}\right]
\end{gathered}
$$

where $\mathrm{m}$ is the body mass; $\left(x_{G}, y_{G}, Z_{G}\right)$ is the center of gravity; $I_{44}, I_{55}$ and $I_{66}$ are the roll, pitch and yaw moments of inertia; the roll-yaw moment of inertia holds the symmetry relation $I_{46}=I_{64} ; A_{w}$ is the water plane area; $M_{w}$ is the first moment of the water plane about the y-axis; $I_{w 1}$ and $I_{w 2}$ are the second moment of the water plane about the $\mathrm{x}$-axis and $\mathrm{y}$-axis respectively; $V$ is the underwater volume; $z_{B}$ is the vertical center of buoyancy.

The standard matrix solution routine provides the complex amplitude of the oscillatory motions from Eq. (27). Then, the wave elevation can be obtained from the dynamic free surface boundary condition in the form

$$
\varsigma=\frac{i \omega_{e}}{g} \sum_{j=0}^{7} \eta_{j} \varphi_{j}+\frac{1}{g} \nabla\left(\varphi_{s}+u_{0} x\right) \cdot \sum_{j=0}^{7} \nabla\left(\eta_{j} \varphi_{j}\right)
$$

\section{Numerical implementation}

\subsection{Discretization of the boundary integral}

In the numerical study, the boundary is divided into a number of quadrilateral panels with constant source density $\sigma(\vec{\xi})$, where $\vec{\xi}=(\xi, \eta, \varsigma)$ is the position vector on the boundary. If $\vec{x}=(x, y, z)$ is inside the fluid domain or on the boundary surface, the potential can be expressed by a source distribution on the boundary of the fluid domain: 


$$
\varphi(\vec{x})=\iint_{S_{b}+S_{f}+S_{c}} \sigma(\vec{\xi}) G(\vec{x}, \vec{\xi}) d S_{\xi}
$$

where $\varphi$ denotes the steady potential $\varphi_{s}$ or the unsteady potential $\varphi_{j}, G(\vec{x}, \vec{\xi})$ is the Rankine-type Green function given by

$$
G(\vec{x}, \vec{\xi})=\frac{1}{\sqrt{(x-\xi)^{2}+(y-\eta)^{2}+(z-\varsigma)^{2}}}
$$

If we have $N$ panels on the body surface, free surface and control surface together, the potential in point $\vec{x}$ becomes

$$
\varphi\left(\vec{x}_{i}\right)=\sum_{j=1}^{N} \frac{\sigma_{j}}{4 \pi} \iint_{S_{b}+S_{f}+S_{c}} G\left(\vec{x}_{i}, \vec{\xi}\right) d S_{\xi}=\sum_{j=1}^{N} \frac{\sigma_{j}}{4 \pi} G_{i, j}
$$

When the collocation point and the panel are close to each other, the influence coefficients $G_{i j}$ can be calculated with analytical formulas listed by Prins (1995) when the distance between the collocation point and the panel is large, these coefficients are calculated numerically. The same procedure can be applied to discretize the boundary integral for the velocity

$$
\frac{\partial \varphi}{\partial n}\left(\vec{x}_{i}\right)=\frac{1}{2} \sigma_{i}+\sum_{\substack{j=1 \\ j \neq i}}^{N} \frac{\sigma_{j}}{4 \pi} \iint_{S_{b}+S_{f}+S_{c}} \frac{\partial}{\partial n_{i}} G\left(\vec{x}_{i}, \vec{\xi}\right) d S_{\xi}=\frac{1}{2} \sigma_{i}+\sum_{\substack{j=1 \\ j \neq i}}^{N} \frac{\sigma_{j}}{4 \pi} G_{i, j}^{n}
$$

The analytical formulas of the influence coefficients $G_{i, j}^{n}$ are listed by Hess and Smith (1964).

\subsection{Desingularied method}

The singularity distribution does not have to be located on the free surface itself, it can also be located at a short distance above the free surface, as long as the collocation points, where the boundary condition has to be satisfied, stay on the free surface. In practice, a distance of maximal three times the longitudinal size of a panel is possible (Bunnik, 1999). In the present study, the raised distance $\Delta z_{i}=\sqrt{S_{i}}$, where $S_{i}$ is the area of the $i$-th panel.

Special attentions should be paid on the second derivative of the potential on the free surface. Generally, the difference schemes can be divided in two classes: up wind difference schemes and central difference schemes. Although central difference schemes are supposed to be more accurate, the stabilizing properties of the upwind difference schemes are more desired in the forward speed problem (Bunnik, 1999). Physically this can be explained by the face that new information on the wave pattern mainly comes from the upstream side, especially at high speeds, whereas the downstream side only contains old information. The first-order upwind difference scheme for the second derivative of the potential to $\mathrm{x}$ can be written as follows 


$$
\frac{\partial^{2} \varphi}{\partial x^{2}}\left(\vec{x}_{i}\right)=\frac{1}{\Delta x^{2}}\left[\varphi\left(\vec{x}_{i+2}\right)-2 \varphi\left(\vec{x}_{i+1}\right)+\varphi\left(\vec{x}_{i}\right)\right]
$$

\section{Validation and application}

In the numerical study, a Wigley III hull advancing in head sea is modelled by using the new radiation condition. Journee's (1992) experimental results are quoted to verify the efficiency of the present model. The model can be defined as

$$
y=\frac{B}{2}\left[1-\left(\frac{z}{D}\right)^{s}\right]\left[1-\left(\frac{2 x}{L}\right)^{2}\right]\left[1+0.2\left(\frac{2 x}{L}\right)^{2}\right]
$$

The main dimensions of Wigley III model is shown in Table 1.

Table 1 Main dimensions of Wigley III hull

\begin{tabular}{lr}
\hline Length, $L(m)$ & 3 \\
Breadth, $B(m)$ & 0.3 \\
Draught, $D(m)$ & 0.1875 \\
Displacement, $V\left(m^{3}\right)$ & 0.078 \\
Centre of rotation above base, $K R(m)$ & 0.1875 \\
Centre of gravity above base, $K G(m)$ & 0.17 \\
Radius of inertia for pitch, $k_{y y}(m)$ & 0.75 \\
\hline
\end{tabular}

The computational range on the free surface is extended to $2 L$ upstream, $2 L$ downstream and $2 L$ sideways, where $L=3 \mathrm{~m}$ is the length of the vessel. Since the flow around a symmetric body is symmetric about $x-Z$ plane, only half computational domain is modelled. There are 300 panels on the body surface, 7200 on free surface and 1200 on the control surface, which is shown in Fig. 8. But the free surface is extended to $1 \mathrm{~L}$ upstream, $3 L$ downstream and $1 L$ sideways in the steady wave problem in order to simulate the full Kelvin wave. 


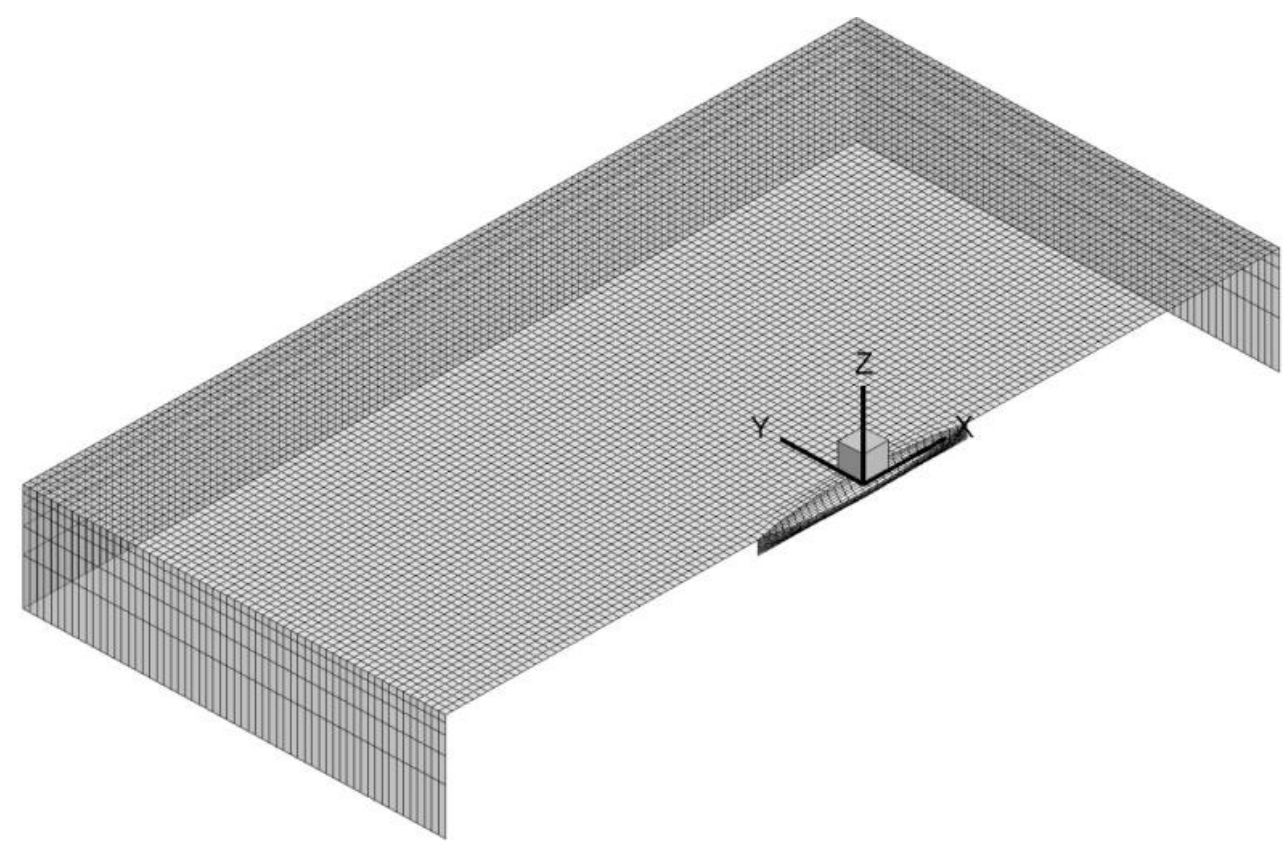

Fig. 8 Panels of the computational model

\subsection{Steady wave problem}
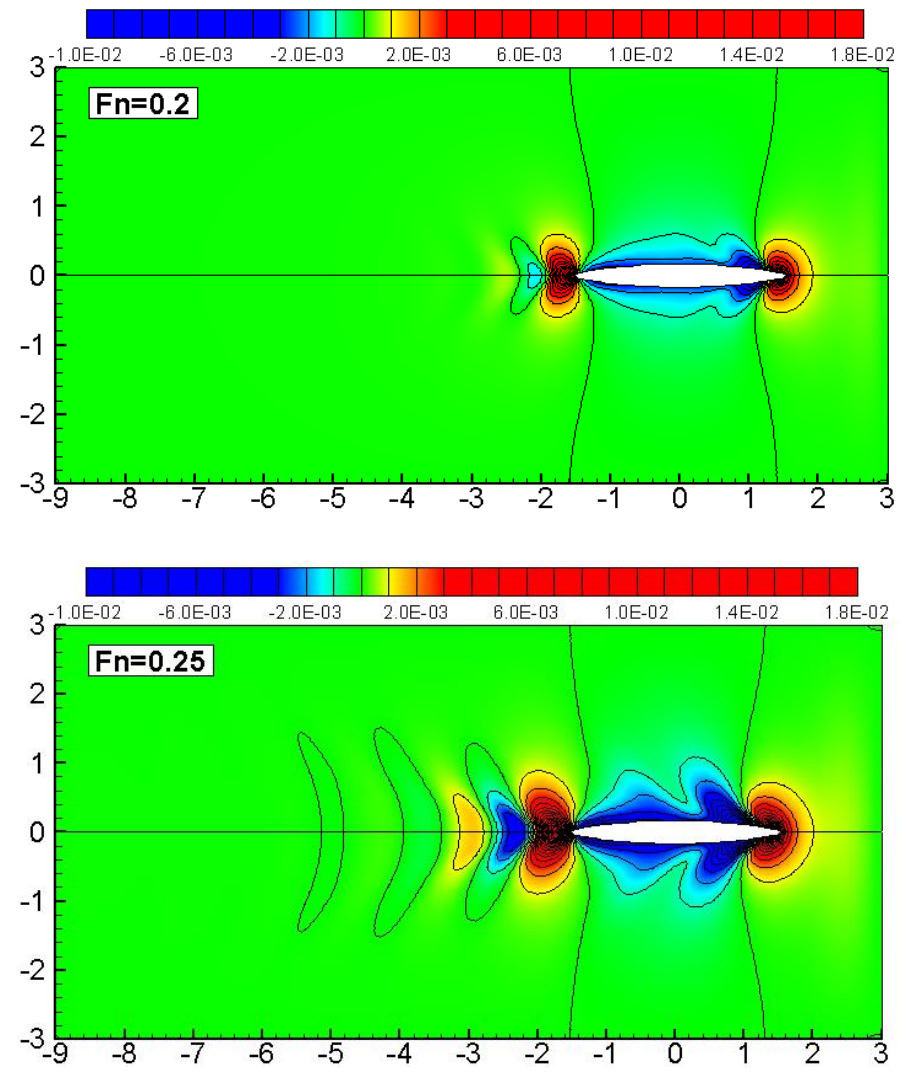

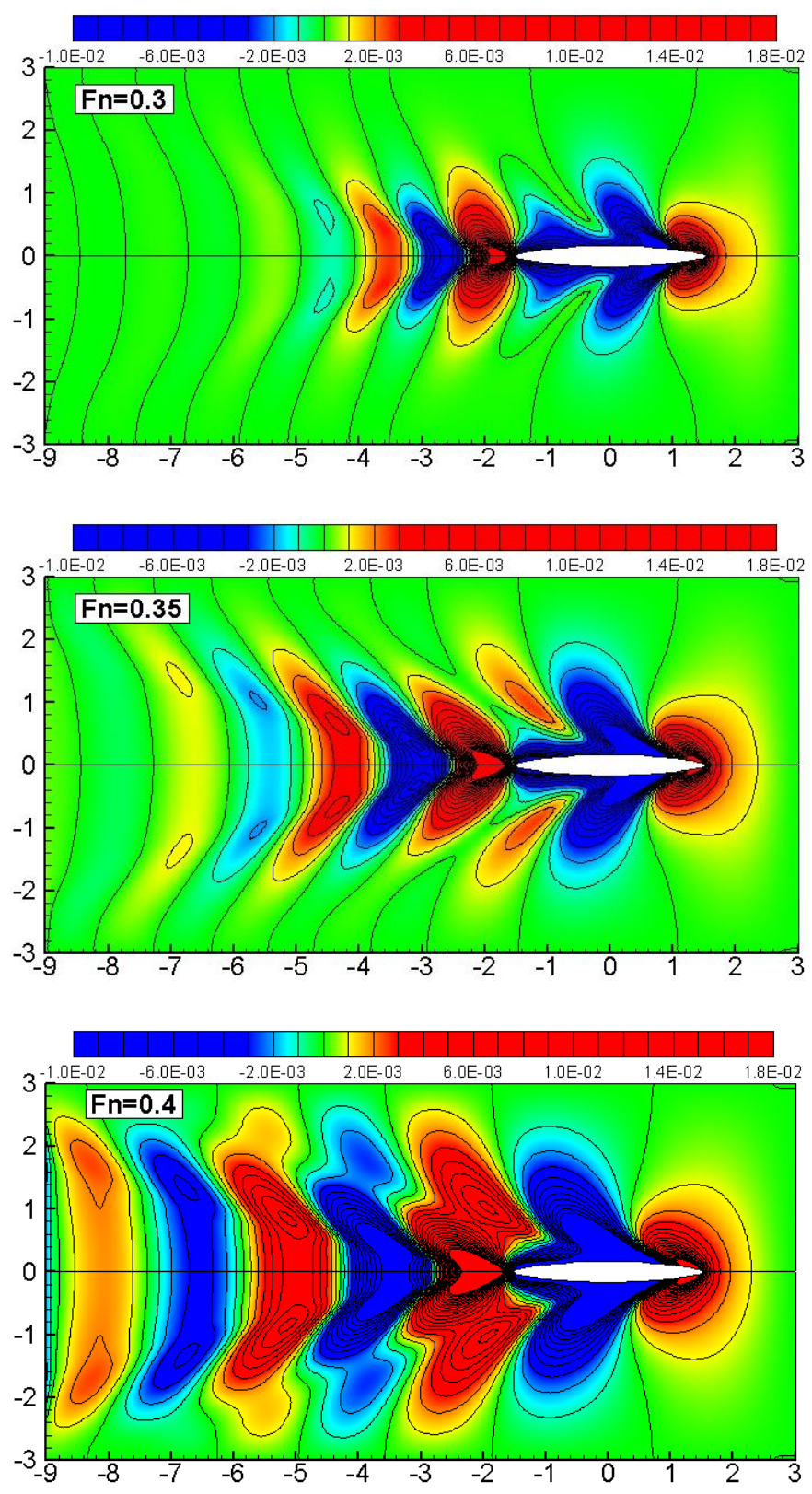

Fig. 9 Steady wave pattern at various speeds 


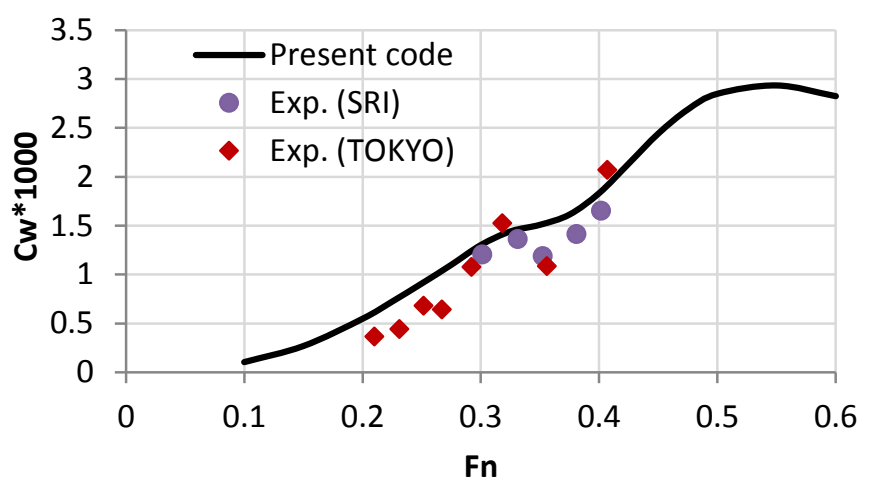

Fig. 10 Wave making resistance of the Wigley hull

Fig. 9 shows the comparison of the wave pattern around the Wigley hull at different Froude numbers $\left(F_{n}=u_{0} / \sqrt{g L}\right)$. As can be seen in these figures, the steady wave elevation attenuates rapidly behind the ship. The diverging waves are radiating from the bow together with the transverse waves following behind the stern. The Kelvin angle increases as the forward speed of the ship increases. At $F n=0.2$, the steady wave elevation is very small, which means the energy dissipation is relatively small at low forward speed. This can also be observed from the wave making resistance in Fig. 10. Compared with the ITTC experimental results (SRI: Ship Research Institute, Tokyo; TOKYO: University of Tokyo), the present results based on the steady flow is satisfactory. It can be found that in the very low forward speed region, the wave making resistance is quite small.

\subsection{The radiation problem}

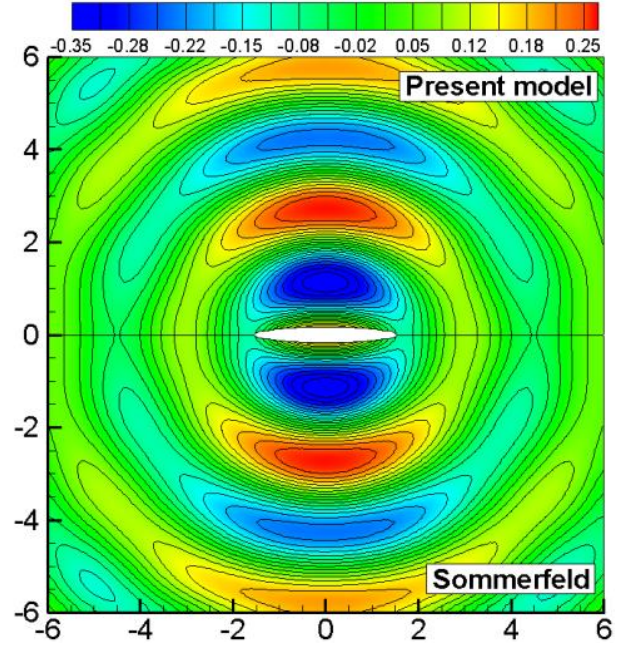

(a) $F n=0, \tau=0$

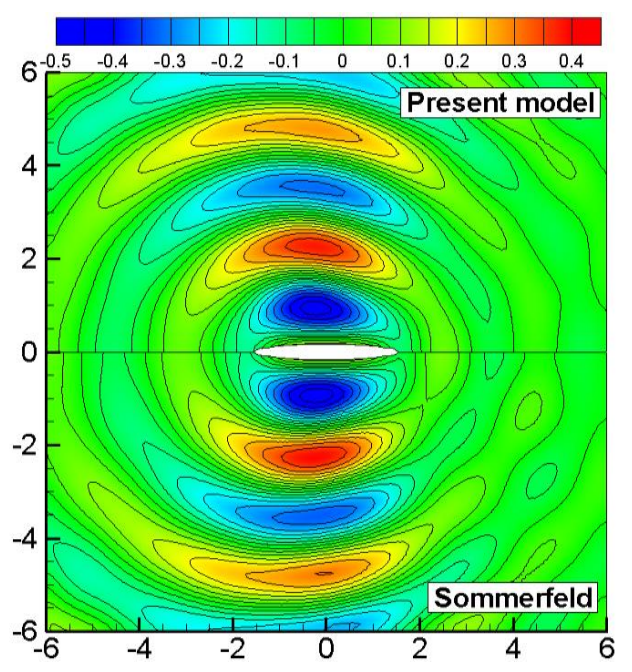

(b) $F n=0.037, \tau=0.1$ 


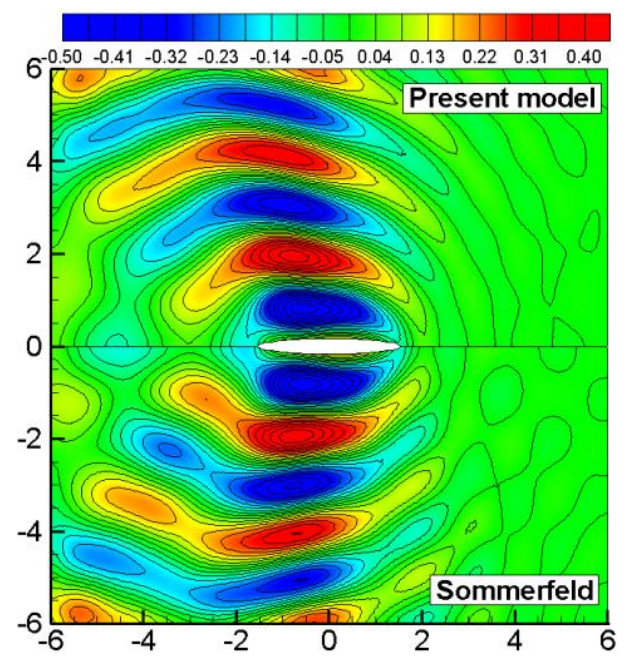

(c) $F n=0.068, \tau=0.2$

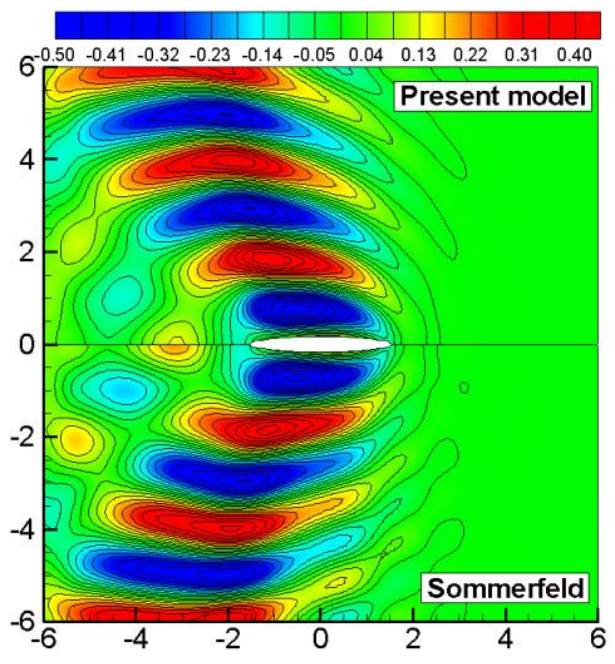

(d) $F n=0.083, \tau=0.25$

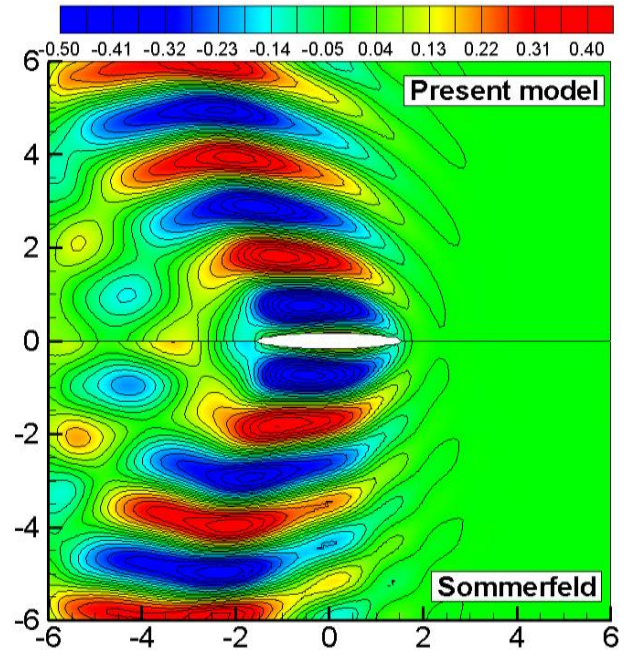

(e) $F n=0.085, \tau=0.26$

Fig. 11 Radiation wave pattern induced by unit heave motion at very low forward speed
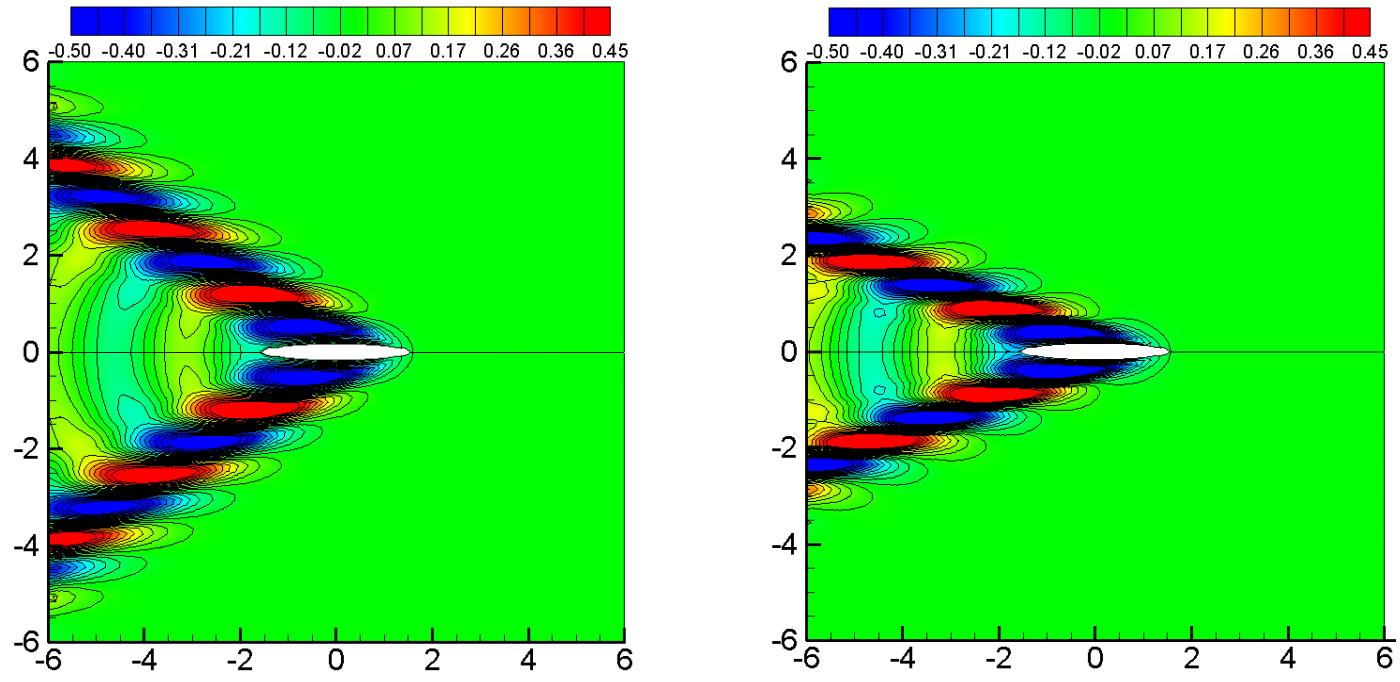

(a) $F n=0.2, \tau=0.75$
(b) $F n=0.3, \tau=1.32$

Fig. 12 Radiation wave pattern induced by unit heave motion at medium forward speed

Fig. 11 and Fig. 12 are the radiation wave pattern induced by unit heave motion. The wave length to ship length ratio of $\lambda / L=1$ in head sea corresponds to the critical conditions in ship design. In order to investigate how the new double Doppler shift radiation condition changes the radiation wave pattern in very low forward speed, we make the comparison between the present model and Sommerfeld radiation condition over various range of Brard number from 0 to 1.32. The lower half of each sub-figure of Fig. 11 shows the wave pattern obtained from Sommerfield radiation condition, while the radiation condition in the upper half account for the new double Doppler shift radiation condition defined by Eqs. (16)-(17). At zero forward speed, the wave elevation calculated from present radiation condition is exactly the same as that from Sommerfeld radiation condition. The waves propagate as a circle pattern beyond one wavelength from the center with the main radiation energy on either side of the vessel. From Fig. 11 (b) and Fig. 11 (c) we can find that even at very low forward speed, the Doppler shift becomes evident and it modifies the wave length. The wave length downstream is larger than the upstream wave length. It can also be found that if the new radiation condition associated with Doppler shift correction is used, the waves appear smooth and stable. While for the Sommerfeld radiation condition, there are some distortions and reflections from the control surface. As the Brard number increases to 0.25 , or just a little greater than 0.25 , the forward speed of the vessel is equal to or greater than the wave group velocity. There should be no waves propagating ahead of the vessel. This phenomenon could be illustrated well from Fig. 11 (d) and Fig. 11 (e). And also, the wave pattern is smooth and stable by using the new model. At $F n=0.2$ and $F n=0.3$, the quiescent region ahead of the vessel expands, and the wave behaves as a $V$-shape downstream, which can be seen from Fig. 12.

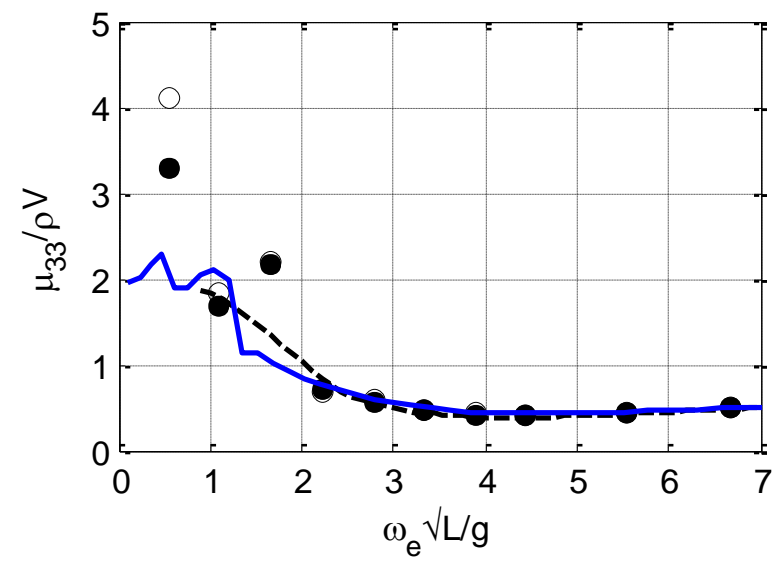

(a) Heave added mass induced by heave motion

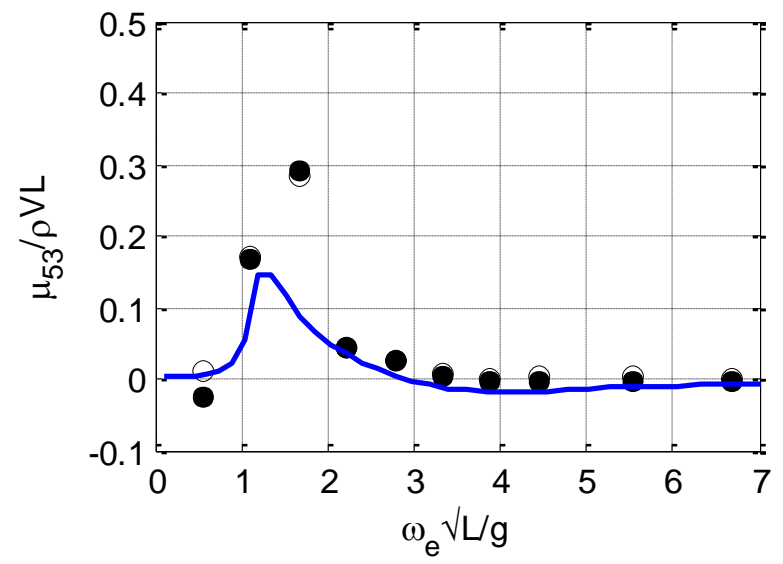

(b) Pitch added mass induced by heave motion 


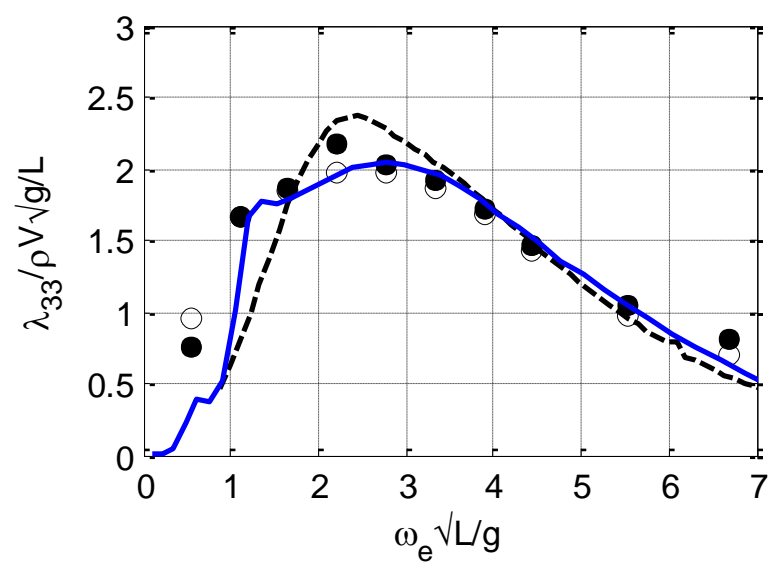

(c) Heave damping induced by heave motion

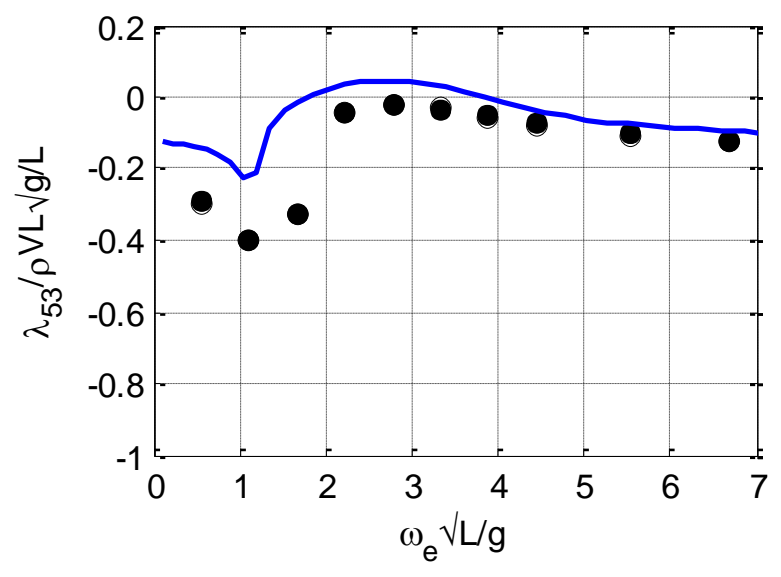

(d) Pitch damping induced by heave motion

Fig. 13 Hydrodynamic coefficients of heave motion at $F n=0.2$. (- -, Fn=0; - , Present calculation; $\circ$, Experiment, $\eta{ }_{3}=2.5 \mathrm{~cm}$; $\bullet$, Experiment, $\eta_{3}=5 \mathrm{~cm}$ )

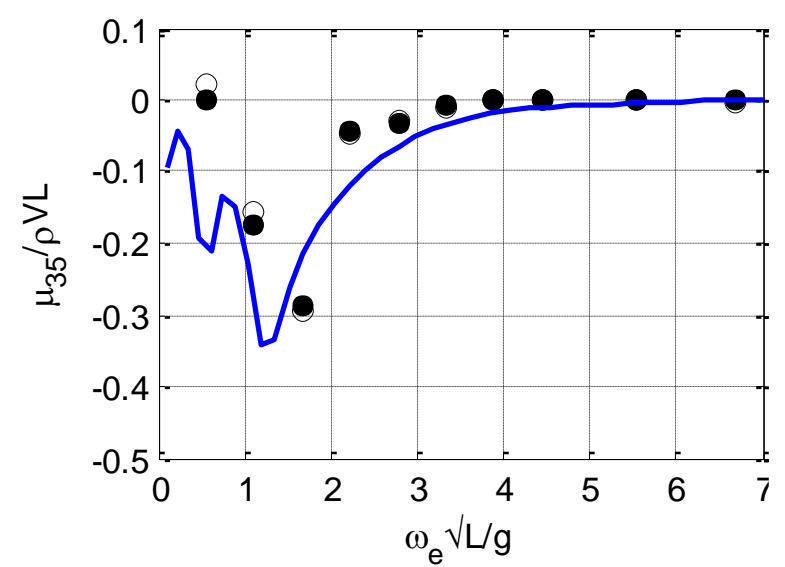

(a) Heave added mass induced by pitch motion

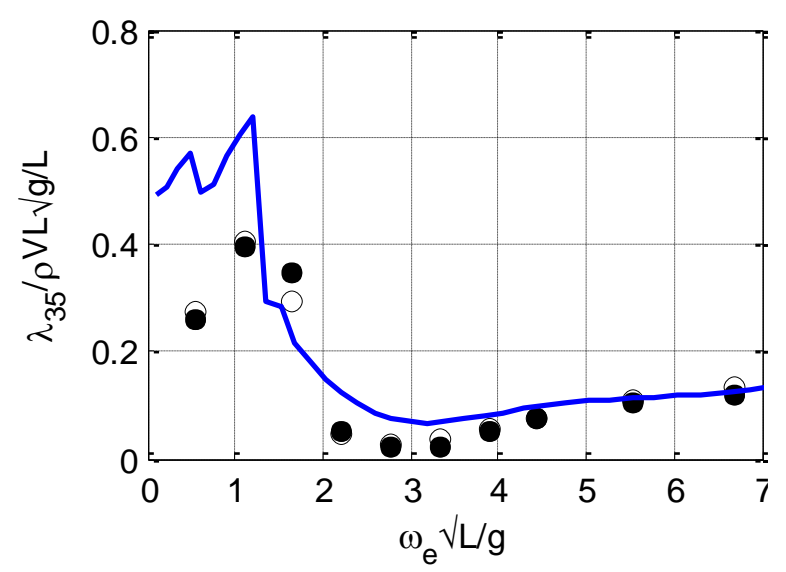

(c) Heave damping induced by pitch motion

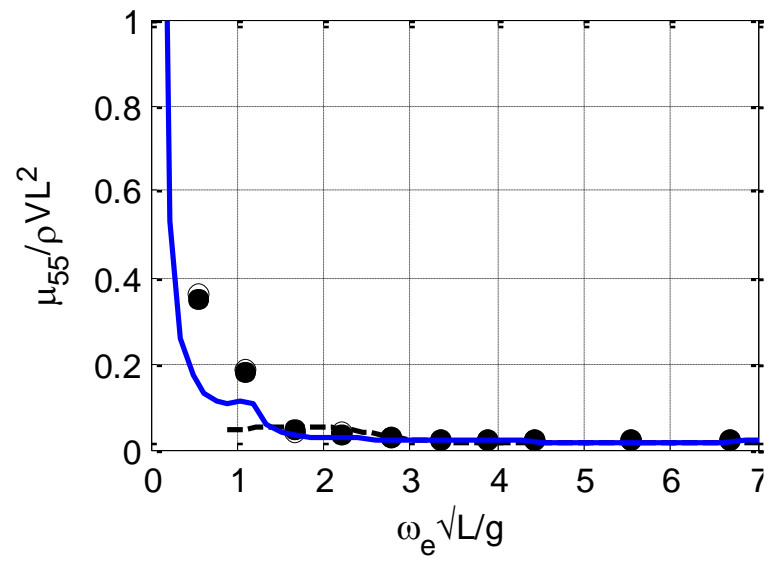

(b) Pitch added mass induced by pitch motion

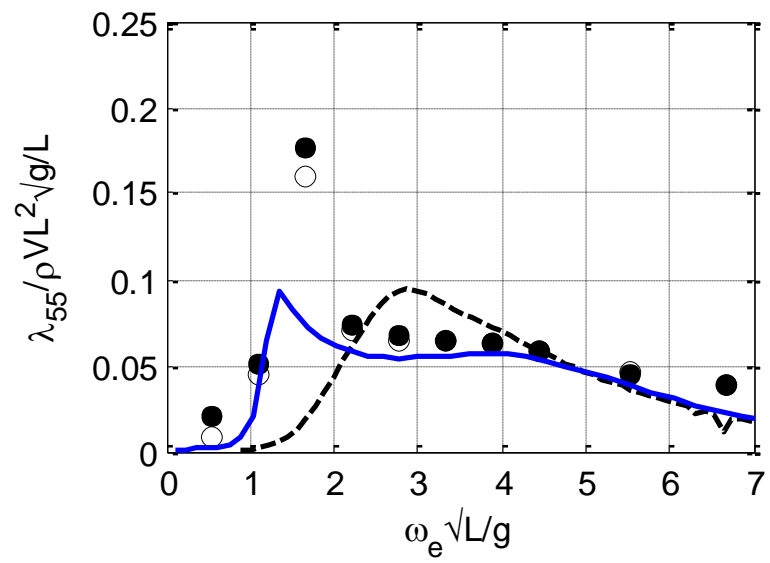

(d) Pitch damping induced by pitch motion

Fig. 14 Hydrodynamic coefficients of pitch motion at $F n=0.2 .\left(--, F n=0\right.$; — , Present calculation; 0 , Experiment, $\eta_{5}=1.5^{\circ}$; $\bullet$, Experiment, $\eta_{5}=3^{\circ}$ ) 
Fig. 13 and Fig. 14 compare the hydrodynamic coefficients between the present calculations and experimental results. Overall, the agreement is quite good in added mass and damping coefficients of heave and pitch motion. Discrepancy arises near $\tau=0.25\left(\omega_{e} \sqrt{L / g}=1.26\right)$. This phenomenon has also been observed by Kim and Shin (2007) by using Green function method. The main reason for the difference lies on the steady wave $m_{j}$ terms given by Eq. (11). In the present code, the free-stream assumption of Eq. (12) is used and the coupling effects between the steady and unsteady flows have been neglected. However, this $m$-term appeared in the body boundary condition will bring some influences to the radiation problem, which is reflected in the hydrodynamic coefficients. In order to get better hydrodynamic coefficients, the double-body or steady-wave flow should be taken as the basic flow. Experimental data for very low forward speed is unfortunately not available.

\subsection{The Diffraction problem}

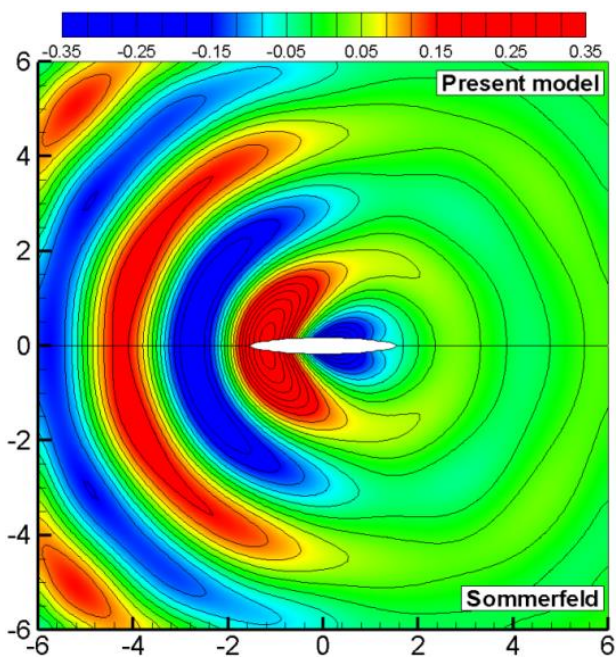

(a) $F n=0, \tau=0$

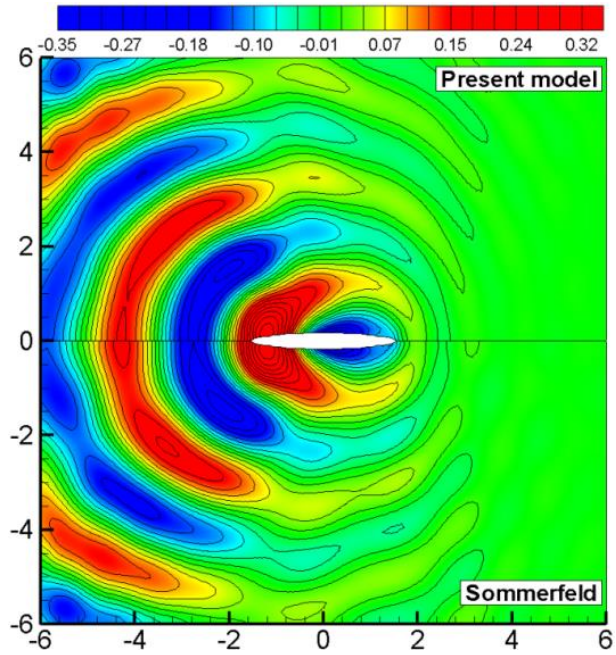

(c) $F n=0.068, \tau=0.2$

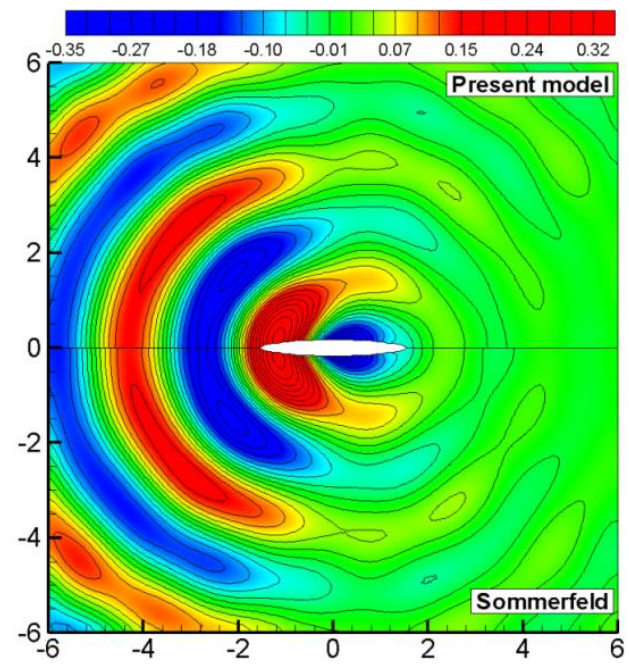

(b) $F n=0.037, \tau=0.1$

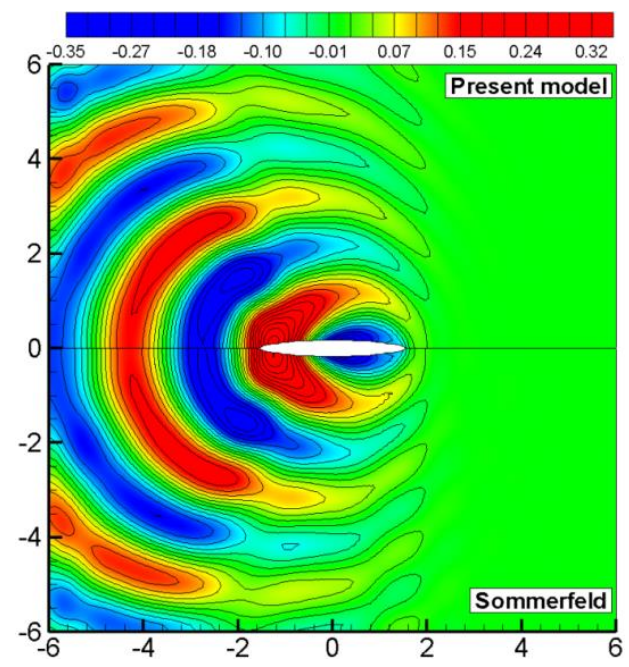

(d) $F n=0.083, \tau=0.25$ 


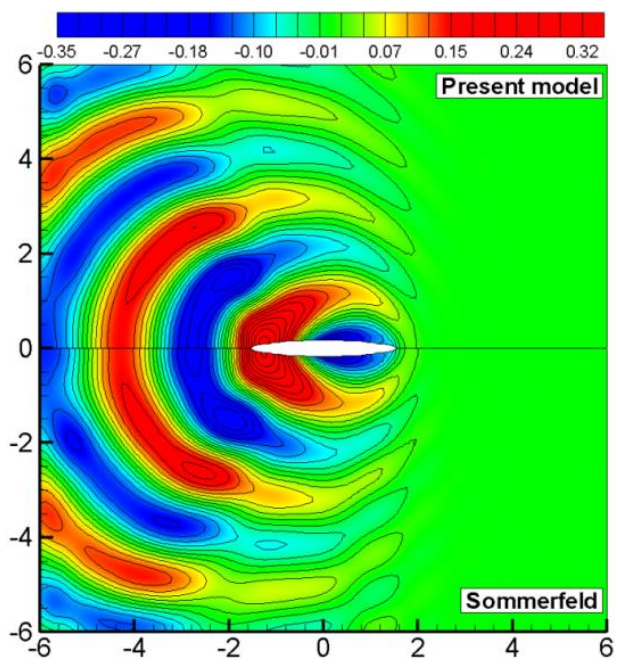

(e) $F n=0.085, \tau=0.26$

Fig. 15 Diffraction wave pattern at very low forward speed

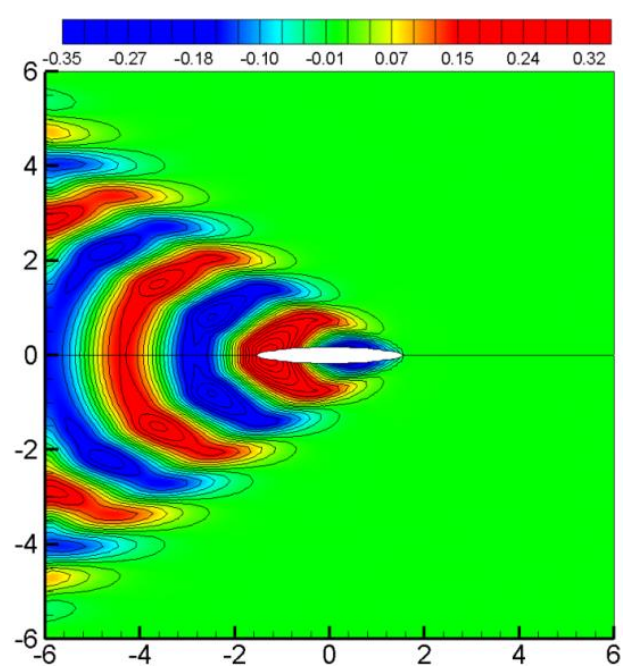

(a) $\mathrm{Fn}=0.2, \tau=0.75$

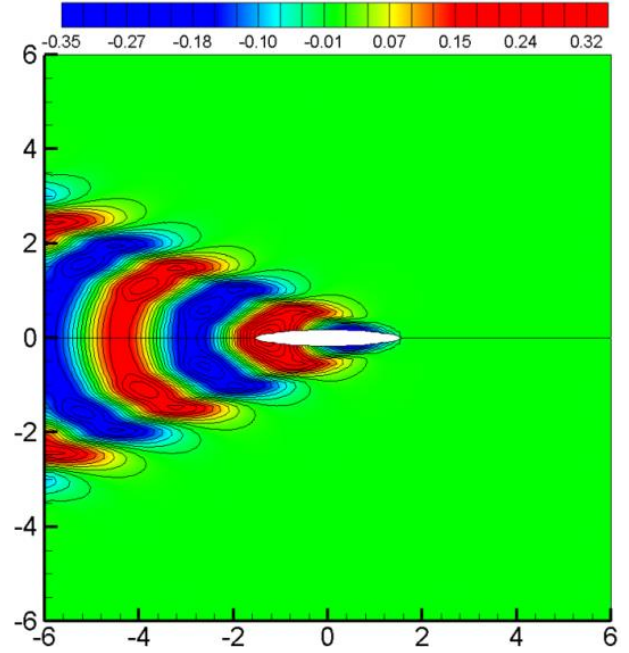

(b) $F n=0.3, \tau=1.32$

Fig. 16 Diffraction wave pattern at medium forward speed

Fig. 15 and Fig. 16 are the diffraction wave patterns induced by unit heave motion. The lower half of each sub-figure of Fig. 15 shows the wave pattern obtained from Sommerfield radiation condition, while the radiation condition in the upper half account for the new double Doppler shift radiation condition defined by Eqs. (16)-(17). At zero forward speed, the two radiation conditions are identical and produce the same wave pattern. But as the Brard number increases up to 0.26, as shown from Fig. 15 (b)-(c), the Doppler shift becomes evident and it modifies the wave length. Some distortions and reflections can be found in the Sommerfeld radiation condition, while the new radiation condition associated with Doppler shift correction provides a better solution of stable wave pattern. Fig. 16 shows the diffraction wave pattern induced by unit heave motion at medium forward speed of $F n=0.2$ and $F n=0.3$. 


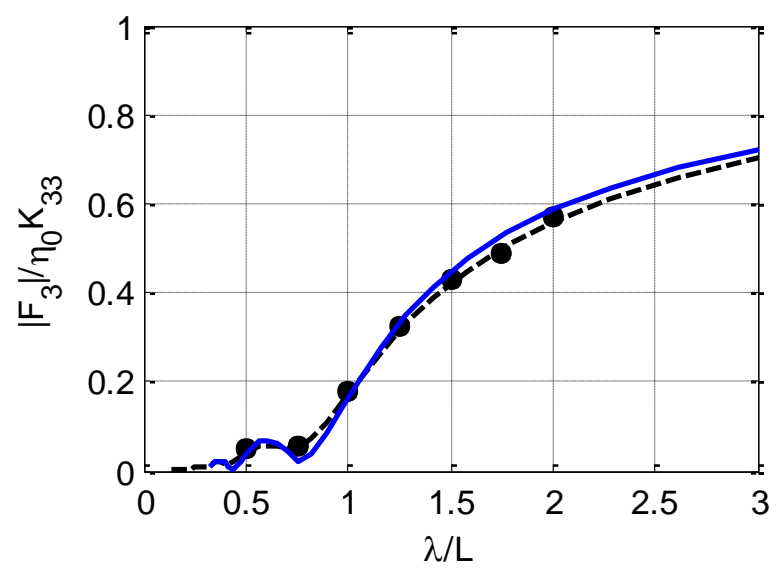

(a)

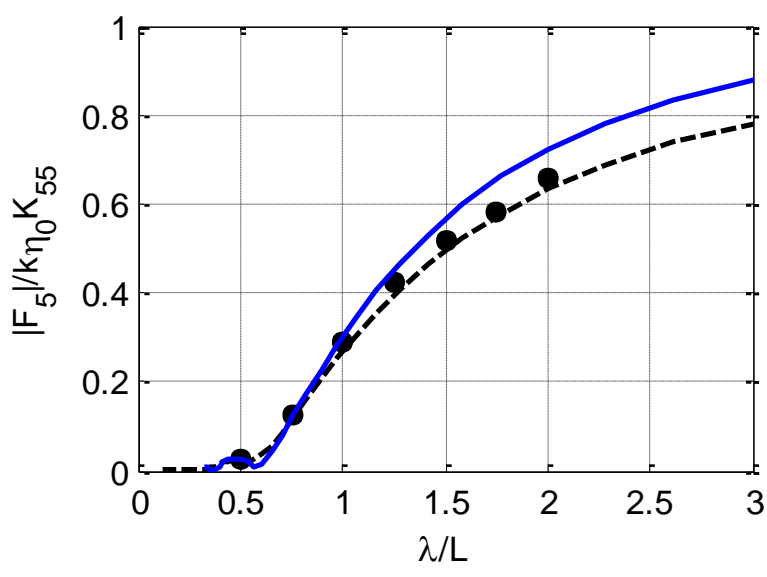

(b)

Fig. 17 Wave exciting forces at $F n=0.2$. (- -, Fn=0; — , Present calculation; $\bullet$ Experiment). (a) Heave; (b) Pitch.

Good agreement of wave exciting forces between the present calculation and experimental results can be observed in Fig. 17. The forward speed effect on the wave exciting forces is not significant. And it is different to the radiation problem that the diffraction potential does not strongly depend on the steady flow solution since $m_{j}$ terms appear only in the body boundary condition for the radiation problem.

\subsection{Response amplitude operator}

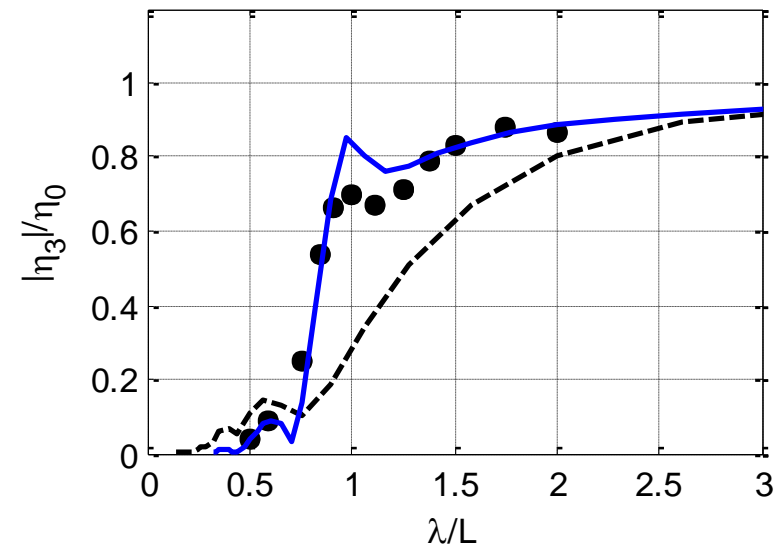

(a)

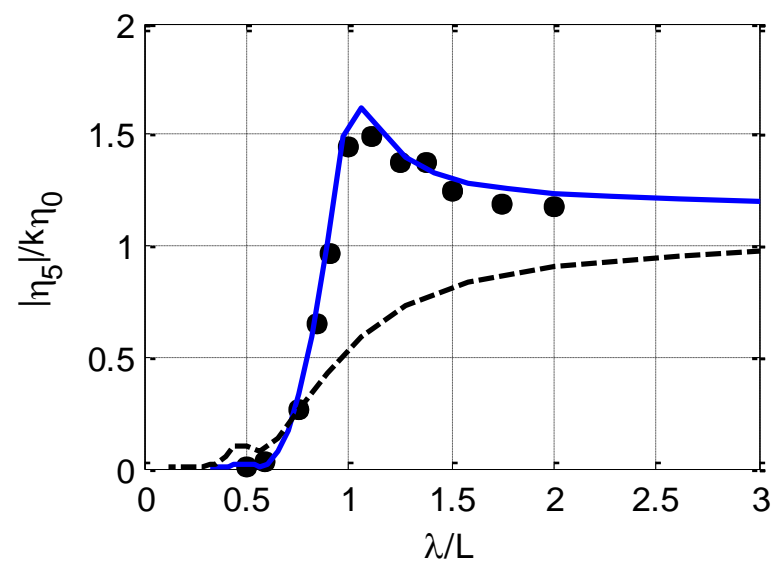

(b)

Fig. 18 Response amplitude operators at Fn=0.2. (- -, Fn=0; -, Present calculation; •, Experiment). (a) Heave; (b) Pitch.

It can be seen from Fig. 18 that the heave and pitch RAO calculated by the present method agrees well with the model test results of Journee (1992). Peak values in the heave and pitch RAOs can be observed at $\lambda / L=1$ and 1.2 corresponding to resonance of the Wigley hull in the respective motions. But at zero forward speed, there is no peak and the RAO curve differs considerably from the forward speed curve.

\section{Conclusions}

A 3-D Rankine source panel method has been developed to predict the hydrodynamic properties of marine vessels travelling with very low or medium forward speeds. A new radiation condition is used when a 
vessel moves with very low forward speed. This new radiation condition corrects the Sommerfeld radiation condition by taking into account of Doppler shift. A Wigley III hull travelling with very low or medium forward speed was considered to verify this radiation conditon. Comparing with the experimental data, the following conclusions can be reached:

1) The new radiation condition associated with Doppler shift correction can provide a good solution of scatterd wave pattern at very low forward speed. The wave field appears more stable and reasonable than Sommerfeld radiation condition over a range of Brard number up to 0.26 .

2) The present method is also effective to predict the hydrodynamic properties of the vessel travelling with medium forward speed. The wave pattern at medium forward speed appears stable and there is no reflection from the control surface. The calculated wave exciting forces and motion RAOs have a very good agreement with the model test results. Small discrepancy appears in predicting the hydrodynamic coefficients in radiation problem due to the coupled effects between the steady flow and radiation potential being neglected, which should be taken into account in the body surface boundary condition in order to obtain more accurate results. Overall, the agreement is found satisfactory.

\section{Acknowledgments}

The present work is mainly funded by Lloyd's Register. Authors are grateful to Lloyd's Register for their support.

\section{References}

Ba, M., Guillbaud, M., 1995. A fast method of evaluation for the translating and pulsating green's function. Ship Technology Research 42 (2), 68-81.

Bunnik, T., 1999. Seakeeping calculations for ships, taking into account the non-linear steady waves, PhD thesis. Delft University of Technology, The Netherlands.

Coudray, T., Le Guen, J.F., 1992. Validation of a 3-D Sea-Keeping Software, 4th CADMO, Madrid, pp. 443-460.

Das, S., Cheung, K.F., 2012a. Hydroelasticity of marine vessels advancing in a seaway. Journal of Fluids and Structures 34, 271-290.

Das, S., Cheung, K.F., 2012b. Scattered waves and motions of marine vessels advancing in a seaway. Wave Motion 49 (1), 181-197.

Gao, Z., Zou, Z., 2008. A NURBS-based high-order panel method for three-dimensional radiation and diffraction problems with forward speed. Ocean Engineering 35 (11-12), 1271-1282.

Hess, J.L., Smith, A.M.O., 1964. Calculation of nonlifting potential flow about arbitrary three-dimensional bodies. Journal of Ship Reaearch 8 (2), 22-44.

Jensen, G., Mi, Z.X., Söding, H., 1986. Rankine source methods for numerical solutions of steady wave resistance problem, Proceedings of 16th Symposium on Naval Hydrodynamics, Berkeley, pp. 575-582.

Journee, J.M.J., 1992. Experiments and calculations on 4 Wigley hull forms in head waves, Report No. 0909. Ship Hydromechanics Laboratory, Delft University of Technology, The Netherlands.

Kim, B., Shin, Y., 2007. Steady flow approximations in 3-D ship motion calculation. Journal of Ship Reaearch 51 (3), 229-249.

Kring, D.C., 1994. Time domain ship motions by a three-dimensional Rankine panel method, PhD Thesis. MIT.

Lee, C.H., Sclavounos, P.D., 1989. Removing the irregular frequencies from integral equations in wave-body interactions. Journal of Fluid Mechanics 207, 393-418. 
Nakos, D.E., 1990. Ship wave patterns and motions by a three dimensional Rankine panel method, PhD Thesis. MIT.

Nakos, D.E., Sclavounos, P.D., 1990. Steady and unsteady ship wave patterns. Journal of Fluid Mechanics 215, 263-288.

Newman, J.N., 1985. Algorithms for the Free-Surface Green Function. Journal of Engineering Mathematics 28 (3), 57-67.

Newman, J.N., 1992. The Approximation of Free-Surface Green Function, Wave Asymptotics, in: Martin, P.A.a.W., G.R. (Ed.), The Proceedings of the Meeting to Mark the Retirement of Professor Fritz Useel from the Beyer Chair of Applied Mathematics in University of Manchester. Cambridge University Press, pp. 107135.

Noblesse, F., Hendrix, D., 1992. On the theory of potential flow about a ship advancing in waves. Journal of Ship Reaearch 36 (1), 17-30.

Nossen, J., Grue, J., Palm, E., 1991. Wave forces on three-dimensional floating bodies with small forward speed. Journal of Fluid Mechanics 227, 135-160.

Prins, H.J., 1995. Time domain calculations of drift forces and moments, PhD Thesis. Delft University of Technology, The Netherlands.

Scalvounos, P.D., Nakos, D.E., 1988. Stability analysis of panel methods for free surface flows with forward speed, 17th Symposium on Naval Hydrodynamics, Den Hague, Nederland. 